\title{
Fatigue strength evaluation for aluminium alloy based on dissipated energy Measurement
}

\author{
by D. Shiozawa*, I. Mishima*, K. Chinzaka*, T.Sakagami* \\ *Affiliation 1, Kobe University, Department, 1-1, Rokkodai, 657-8501, Kobe, Japan, shiozawa@mech.kobe-u.ac.jp
}

\begin{abstract}
Fatigue limit estimation based on the dissipated energy measurement has been getting an attention in various industries because of its time and cost effectiveness compared to S-N curves. The effectiveness of this method has been reported for steels. In this study, the dissipated energy for aluminum alloy was measured and the applicability of the fatigue strength estimation based on the dissipated energy was discussed. The dissipated energy for aluminum alloy A5052 specimen was measured in stair case like stress level test. It was found that the dissipated energy increases with increasing stress levels, and a certain stress level where the change in dissipated energy $\Delta T_{\mathrm{D}}$ shows sharp increase coincides with fatigue limit obtained from S-N curves. The change of dissipated energy for A5052 is similar to that for steels, on the other hand, it was different from that for aluminum alloy A6061 which have no fatigue limit.
\end{abstract}

\section{Introduction}

Fatigue limit rapid estimation method has been studied by several researchers. This method can obtain the fatigue limit of material from the short-term fatigue tests of few specimens, so that this method has time and cost advantage. The effectiveness of this method has been reported for steels. Due to the demand for longer life, lower cost, and lighter weight of products, the aluminum alloy is used as a structural member for vehicles and ships. It is useful if fatigue strength can be evaluated quickly by measuring dissipative energy similarly for A5052. This paper describes the results of energy dissipation measurements for the A5052-O.

\section{Dissipated energy and fatigue limit estimation}

It is known as thermoelastic effect that temperature rise is observed under the application of compressive stress, and temperature fall is observed under the application of tensile stress (Fig. 1). Thermoelastic temperature change $\Delta T_{\mathrm{E}}$ is formulated by thermoelastic coefficient $k$, absolute temperature $T$, and sum of principal stresses $\Delta \sigma$ as follows.

$$
\Delta T_{\mathrm{E}}=k T \Delta \sigma
$$

Under cyclic loading, temperature rise due to irreversible energy dissipation is observed in addition to the thermoelastic temperature change. This kind of heat generation is called dissipated energy and is caused by local plastic deformation at the maximum tensile stress and at the maximum compressive stress. Temperature change due to dissipated energy $\Delta T_{\mathrm{D}}$ can be measured by lock-in algorism using basically double frequency for the loading frequency.

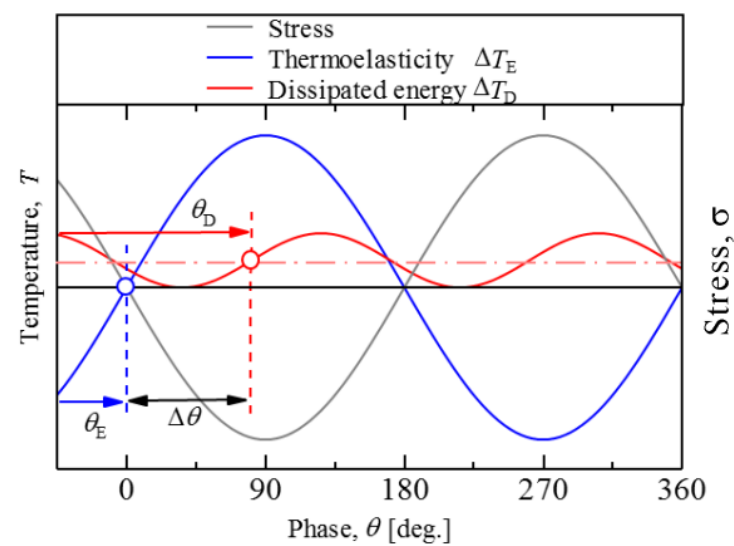

Fig. 1 schematic illustration of temperature change and phase difference.

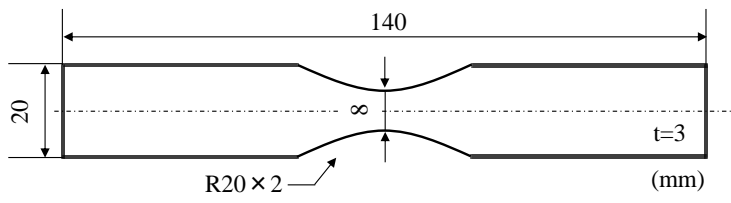

Fig. 2 Geometry of specimen 


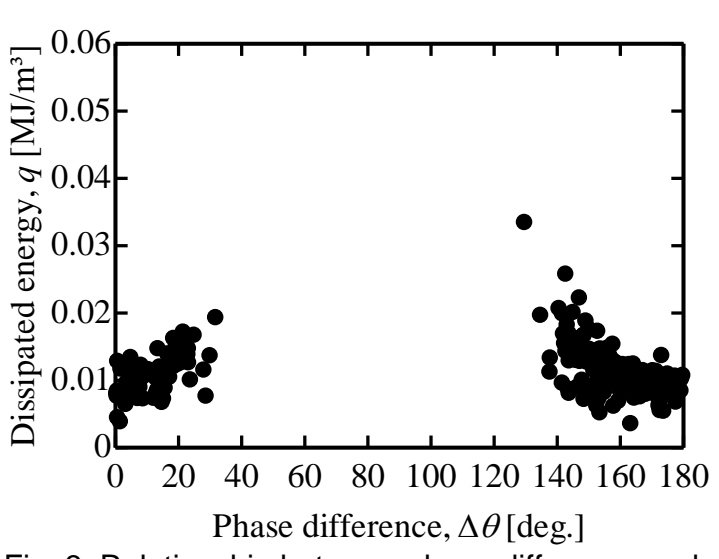

Fig. 3 Relationship between phase difference and dissipated energy. $\left(\sigma_{\mathrm{a}}=160 \mathrm{MPa}\right)$

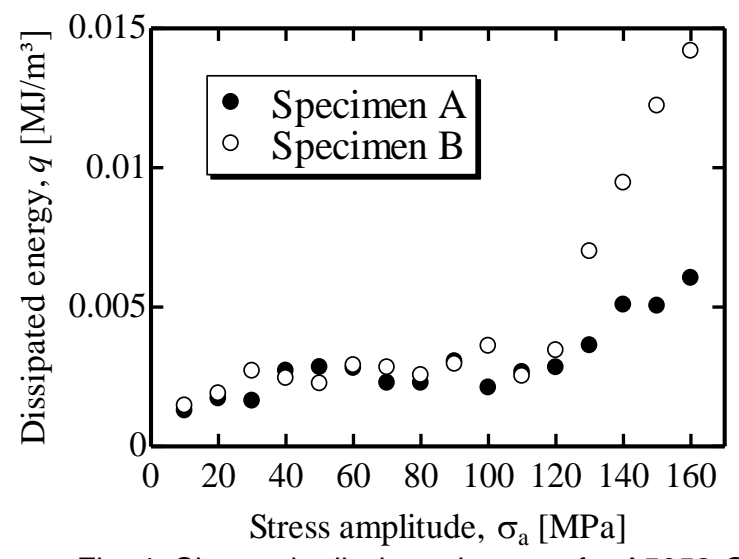

Fig. 4 Change in dissipated energy for A5052-O

In the fatigue limit estimation based on energy dissipation, temperature rise due to irreversible energy dissipation is measured by infrared thermography for various levels of stress amplitude. Usually staircase-like stress level test is carried out for obtaining temperature rise due to dissipated energy for each stress level. Then, temperature rise due to dissipated energy $\Delta T_{\mathrm{D}}$ increases with increasing stress levels, and the bilinear relationship is observed. It is known that the stress level $\sigma_{w}^{\prime}$ where the change in $\Delta T_{\mathrm{D}}$ shows sharp increase coincides with fatigue limit $\sigma_{\mathrm{w}}$ obtained by conventional fatigue test based on 10 million stress cycles.

\section{Experimental results}

\subsection{Materials and methods}

In this study, staircase-like stress level test was carried out for A5052-O aluminum alloy. Geometry of the specimen is shown in Fig. 2. The specimen has smooth notches with stress concentration factor $\alpha$ of 1.03. Fully reversed cyclic axial loading (stress ratio $R=-1$ ) with a frequency of $7 \mathrm{~Hz}$ was applied to the specimen. In the staircase-like stress level test, stress level was changed from $10 \mathrm{MPa}$ to $160 \mathrm{MPa}$. Dissipated energy emitted from specimen surface in fatigue test was measured by infrared thermography with a MCT array detector (CEDIP Inc. Titanium, detectable wavelength: 7.7$9.3 \mu \mathrm{m}, \mathrm{NETD}: 25 \mathrm{mK})$.

\subsection{Experimental result and consideration}

The phase difference between the thermoelastic temperature change and temperature change due to the energy dissipation exits in the measured temperature change. This time difference indicates the timing of heat generation due to the plastic deformation. Figure 3 shows the relationship between the dissipated energy and its phase difference. From Fig. 3, it can be seen that there are two peak of phase difference at 30 degrees and 150 degrees. In the previous studies, it has been reported that the phase difference near 150 degrees contains the information due to the noise caused by the harmonic component of the fatigue testing machine. In order to improve the dissipated energy measurement, the data analysis processing using phase information (phase $2 f$ lock-in technique) was performed to remove the influence of noise component due to the fatigue testing machine [1].

Fig. 4 shows the change in the dissipated energy at the center of specimen. It is found from Fig. 4 that the dissipation energy increases from $\sigma_{\mathrm{a}}=120 \mathrm{MPa}$. The change of dissipated energy for A5052 is not similar to that for A6061-T6 aluminum alloy [2] which don't have fatigue limit, and it is similar to that for austenitic stainless steel type 316L. The fatigue limit of A5052-O is about $120 \mathrm{MPa}$, so the stress amplitude where the dissipated energy increase coincides with the fatigue limit of A5052-O.

\section{REFERENCES}

[1] D. Shiozawa, T. Inagawa, T. Washio, T. Sakagami, Accuracy improvement in dissipated energy measurement by phase information, measurement science and technology, Vol. 28(2017).

[2] A. Akai, D. Shiozawa, S. Funazo, K. Chinzaka and T. Sakagami, "Fatigue strength evaluation for aluminium Alloy A6061-T6 based on dissipated energy measurement, Journal of the Society of Materials Science, Japan, Vol. 67, No. 12(2018), pp. 1036-1041. 